\title{
Der Wandel des Medienangebots als Herausforderung an die Journalismusforschung: Plädoyer für eine kulturorientierte Annäherung
}

\author{
Elisabeth Klaus / Margret Lünenborg
}

Der Aufsatz setzt sich kritisch mit zentralen theoretischen Prämissen der aktuellen Journalismusforschung auseinander. In der Konzentration auf den nachrichtlichen Journalismus einerseits und die Kommunikatoren andererseits gelingt es der Journalistik nicht, aktuelle Veränderungen angemessen zu beschreiben und analytisch zu erfassen. Anstelle der Paradigmen "System “versus „Individuum “ wird hier das Paradigma „Kultur" als erkenntnisleitende Perspektivierung eingesetzt. Auf Basis der Cultural Studies können vor allem auch die populären Formen des Journalismus und seine Publikumsorientierung integrativ untersucht werden. Information und Unterhaltung werden dann nicht länger als funktionaler Gegensatz konstruiert. Zugleich gelingt es mit dem Kreislauf medialer Produktion und Rezeption, den gesellschaftlichen Prozess der Sinnvermittlung durch Journalismus transparent zu machen und damit Forschungsaufgaben für eine kulturorientierte Journalistik zu formulieren.

Gerhard Schröder, der „Medienkanzler“, präsentiert politisches Handeln als medienöffentliches Theater und weiß dabei die symbolische Ordnung der Macht neu zu gestalten. Er demonstriert auf diese Weise eine bemerkenswerte Souveränität im praktischen Umgang mit jenen Veränderungen, Verschiebungen und Neuorientierungen im Journalismus, mit deren Verständnis sich die Journalismusforschung noch schwer tut. Die Choreographie des Parteitagsauftakts lehnt sich - mit Blick auf die laufenden Fernsehkameras - an den Einmarsch von Box-Champions an. Die politische Berichterstattung regionaler wie überregionaler Medien beschäftigt sich mit den Kleidungs-, Ess- und Rauchgewohnheiten des Kanzlers. Als Kanzlerkandidat spielte Schröder sich selber in der erfolgreichsten deutschen Soap-Opera "Gute Zeiten - Schlechte Zeiten“ (RTL) und reihte sich damit in die Reihe jener Politiker ein, die populäre Genres zur Erhöhung der eigenen Popularität zu nutzen versuchen. Politiker scheinen die Stars eines neuen grenzverletzenden Genres zu sein, der „Polit-Soap “ 1 , die die wohl vertraute Einteilung der Medienproduktion in fiktionale und nichtfiktionale Inhalte sprengt, weil Politik gefällig als Seifenoper inszeniert und Soaps zur Verbesserung des Images genutzt werden. Politik mittels Unterhaltung? Unterhaltung durch Information? All das wirft die Frage nach der Scheidelinie zwischen Journalismus und Banalität, zwischen Öffentlichkeit und Populismus, zwischen Seriösem und Boulevardeskem auf. Sind solche Unterscheidungen überhaupt noch möglich und sind sie in ihrer dualistischen Struktur sinnvoll? Tragen sie zum Verständnis der aktuellen Erscheinungen des Journalismus bei?

Sicher ist allein, dass sich die Journalismusforschung mit solchen Fragen beschäftigen muss, will sie die seit Mitte der 80er Jahre stattfindenden Umbrüche in der Medienlandschaft und die neueren Entwicklungen im Journalismus verstehen. Mit den zentra-

1 Göttlich und Nieland (1997) haben mit diesem Ausdruck Entwicklungen im Nachrichtengenre gekennzeichnet. 
len Stichworten der Debatte, „Personalisierung “, „Privatisierung“, „Entpolitisierung “2, „Fiktionalisierung“ (Mettler-v. Meibom 1996) oder „Boulevardisierung“ (Krüger 1996) werden Phänomene benannt, deren Durchdringung eine Herausforderung an die aktuelle Journalismusforschung darstellt. Jedoch zeigen sich genau an dieser Aufgabenstellung methodische und empirische Defizite der Journalismusforschung, die bisher vor allem auf die beiden von Rühl (2000) identifizierten Paradigmen - „Subjekt“ und „System" - konzentriert ist. Die Leerstellen verweisen auf die Notwendigkeit einer theoretischen und empirischen Neuorientierung, die mit Hilfe einer kulturorientierten und handlungstheoretischen Perspektivierung der Journalistik erfolgen müsste. Die Cultural Studies bieten dafür einen geeigneten theoretischen Rahmen.

Im Folgenden wollen wir darstellen, welche Beschränkungen mit den empirischen Schwerpunkten und den dominierenden theoretischen Prämissen der deutschen Journalismusforschung verbunden sind. Zentral erscheinen uns dabei zwei Problemfelder: Einerseits die weitgehende Gleichsetzung von Journalismus mit Nachrichten- und Informationsjournalismus, die eine Vernachlässigung anderer journalistischer Arbeitsweisen, Funktionen und Präsentationsweisen bedingt. Diese Verengung führt andererseits dazu, dass der gesellschaftliche Prozess, in den die Produktion von Medieninhalten eingebunden ist, sowie das Publikum und seine Weise, sich journalistische Texte anzueignen und mit Bedeutung zu versehen, randständig geblieben sind.

\section{Unterhaltung als konstitutive Funktion des Journalismus}

Dem kritischen Blick von Schriftstellern und Medienkritikern hielten sie nicht stand, Medienwissenschaftlern waren sie ein Dorn im Auge, Zeitgenossen und Medienhistoriker waren sich in ihrem Urteil über die moralischen Wochenschriften des 18. Jahrhunderts einig: minderwertige Elaborate, langweilig, banal, unpolitisch! Die moralischen Wochenschriften versuchten, auf unterhaltsame Weise Orientierung, Alltagshilfe und Bildung zu vermitteln. Heute würden sie als Ratgeberjournalismus klassifiziert. Mit dem Abschied vom Lateinischen gelang es den Herausgebern, ein breites, vorrangig weibliches Publikum für sich zu gewinnen. Lesen sollte nicht nur bilden, es durfte auch einfach Spaß machen. Die Moraljournalisten - hinter männlichem Pseudonym verbargen sich allerdings auch einige der frühen Journalistinnen - hat Maar (1995) als Väter des Infotainment entdeckt. Mit neuen Genres wie dem (fiktiven) Leserbrief oder der Familienserie entstand eine besondere Nähe zum lesenden Publikum.

Dass mit Schlagworten wie „Personalisierung“, „Privatisierung“ oder „Entpolitisierung“ die Spezifik und die Bedeutung der aktuell stattfindenden Veränderungen im Journalismus nicht hinreichend beschrieben werden kann, zeigt auch Bönings (1998) Fallstudie des frühen Hamburger Pressewesens. Während Mitte des 18. Jahrhunderts selbst in den unterhaltungsorientierten moralischen Wochenschriften das Privatleben konkreter Personen noch tabu war, hielt es bereits in den für das gemeine Volk geschriebenen Zeitungen Einzug und verdrängte zunehmend die personenunabhängige Information. Mit dem Erscheinen der Traueranzeigen Ende des Jahrhunderts hat das Persönliche auch Einzug in die seriösen Zeitungen gehalten. In den zahlreichen Intelligenzblättern ist es traditionell als Mittel der belehrenden Unterrichtung legitimiert und nimmt entsprechend breiten Raum ein. Auf diese Weise, so Böning, reklamiert eine

2 Mit diesen drei Begriffen hat Ross (1998) die Veränderungen im Beziehungsdreieck von Privatheit, Öffentlichkeit und Politik beschrieben. 
Schicht von gebildeten Bürgern ihr Deutungsmonopol in sittlich-moralischen Fragen. „Doch es zeichnet sich bereits $a b$, daß die Veröffentlichung des Privaten nicht nur marktfähig, sondern sogar ein großer Markterfolg ist. Zensur, Staat, Geistlichkeit und bürgerliche Öffentlichkeit wehren sich gleichermaßen gegen diese Zumutung. “ (Böning 1998: 53)

Zusammenfassend liefern beide historischen Studien einige wichtige Erkenntnisse für die Betrachtung des heutigen Journalismus. Erstens: „Unterhaltungsorientierung“ und „Orientierung am Privaten“ unterscheiden die gegenwärtigen Veränderungen im Journalismus nicht deutlich von anderen Zeiten. Zweitens geht die Entwicklung unterhaltender Formen und die Personalisierung von Inhalten mit einer zunehmenden Marktund Publikumsorientierung des Journalismus einher. Drittens nützt die Abwertung und Trivialisierung der populären, unterhaltenden Medienformen dem sich neu formierenden Bürgertum, untermauert also ideologisch den Führungs- und Herrschaftsanspruch einer bestimmten gesellschaftlichen Gruppe. Viertens schließlich geschieht die Abwertung der Unterhaltungskultur im Rückgriff auf die für die Aufklärung konstitutiven Dualismen von öffentlich und privat, politisch und persönlich, rational und emotional, geistig und körperlich. Dabei werden den populären Medienprodukten in der dualistischen Wertehierarchie die jeweils untergeordneten, minderwertigen Positionen zugewiesen: Das Publikum zerfällt in die aufklärungsbedürftige, aktive Bürgerin und den unterhaltungssuchenden, passiven Konsumenten.

Dieser zugleich politisch, philosophisch wie normativ fundierte Standpunkt der Kritiker, von dem aus eine Medienentwicklung hin zu einer stärkeren Publikums- und Alltagsorientierung negativ beurteilt wird, erweist sich seit dem 18. Jahrhundert als erstaunlich stabil. Bis heute wird die Einschätzung des Journalismus und der Medienprodukte nachhaltig vom Aufklärungsmythos beeinflusst, in dem Öffentlichkeit, Politik, Männlichkeit, Aktivität, Geist und Verstand als sich gegenseitig verstärkende Normen miteinander verwoben sind. So erklärt Weischenberg (1997: 11) eine Frau zum Synonym für Qualitätsverlust und Verführung des Publikums:

„Schreinemakerisierung wollen wir den Prozeß nennen, der zu einem neuen ,TVJournalismus' führt - wenn nicht zu einem neuen Journalismus überhaupt. (...) Schreinemakerisierung, das ist die Inszenierung einer permanenten Seifenoper, die als Journalismus verkauft wird. Tatsächlich wird dabei Journalismus durch Entertainment mit stark fiktionalen Elementen ersetzt, ohne daß dies dem Publikum bewußt ist. Dieses Publikum will gewiß immer noch Information und Aufklärung - aber womöglich immer mehr ohne den Aufwand der persönlichen Anstrengung.“

Information und Aufklärung - im Zitat finden sich die zentralen Normen, die der Journalismusforschung als Prüfkriterien zugrunde liegen und die Beurteilung journalistischer Exzellenz und Qualität nachhaltig bestimmen. Deutlich wird diese normative Sicht auch in Blöbaums historischer Skizze der Ausdifferenzierung des Journalismus: „Durch die Vermittlung von Informationen befriedigt Journalismus Wissensdurst und Aufklärungseifer und schafft damit gleichzeitig die notwendige Basis für den aufgeklärten Diskurs." (Blöbaum 1994: 168) Die Verknüpfung von Information und Aufklärung beschreibt die dem Journalismus zugewiesene politische Funktion in der bürgerlichdemokratischen Gesellschaft. Ein Journalismusbegriff, der die Information zum zentralen Merkmal journalistischer Tätigkeit erhebt, grenzt damit zugleich die Unterhaltung aus und nimmt vielerlei Ausschlüsse vor.

Auf Seiten der KommunikatorInnen werden ganze Berufsfelder marginalisiert: Die Tätigkeiten von Sportjournalistinnen und Modezeitschriftenredakteuren, Fachzeitschriftenjournalistinnen und Männermagazinmachern erscheinen für den aufgeklärten 
politischen Diskurs kaum von Belang. Nur vereinzelt und am Rande der Journalismusforschung wurden sie zum Thema (vgl. z. B. Langenbucher/Mahle 1974; Görner 1995; Matthiesen 2000). In Bezug auf die Frage, wer denn nun eigentlich ein Journalist ist, scheint jedenfalls die Kluft immer größer zu werden zwischen einer engen journalistikwissenschaftlichen Bestimmung, die weiterhin um den „Nachrichtenschreiber" (Starkulla 1963) kreist, und einer alltagsweltlichen, derzufolge „jeder und jede - auf dem Niveau herrscht Gleichberechtigung - als Journalist durchgeht, der schon einmal „guten Abend, allerseits" vom Teleprompter abgelesen hat. Wer sein Kinn in die Hand stützen kann, ohne abzurutschen, ist bereits Publizist" (Jürgs 1999: 64). Auf Seiten der Medienangebote ist für große Teile des Zeitschriftenjournalismus, für zahlreiche redaktionell produzierte Hörfunk- und Fernsehsendungen, aber auch für einige Bereiche aus dem Tageszeitungsjournalismus - Sport, Reise, Mode, Vermischtes - in Frage gestellt, ob sie überhaupt zum Journalismus gehören.

Wer aus der Perspektive eines auf die Informationsfunktion und die Aufklärung verkürzten Journalismusbegriffes die Veränderung der Medienlandschaft und die Entwicklung des Journalismus betrachtet, separiert häufig auch das Publikum in ein informationsorientiertes und ein unterhaltungsorientiertes. Für den Rezeptionsprozess ist jedoch empirisch belegt, dass die Trennung zwischen Information und Unterhaltung nicht haltbar ist (Dehm 1984). Rezipienten und Rezipientinnen informieren sich auch aus fiktionalen Unterhaltungsangeboten und sie nutzen Informationsangebote zur Unterhaltung. Aus Sicht des Publikums sind Information und Unterhaltung keine Gegensätze, sondern hier gilt: Der Gegensatz von Information ist Desinformation, der Gegensatz von Unterhaltung ist Langeweile (vgl. Klaus 1996; Bosshart 1999).

Unterhaltung ist kein notwendiges Übel des Journalismus, sondern war für ihn seit seiner Entstehung konstitutiv. Ohne die Wahrnehmung der Unterhaltungsfunktion ist die Herausbildung und Ausdifferenzierung des Journalismus gar nicht denkbar. So zeigt Maar (1995), dass die kurzweilige Präsentationsweise der moralischen Wochenschriften überhaupt erst jene Bindung ermöglichte, die notwendig war, um ein Massenpublikum zu erreichen (vgl. auch Böning 1998). Offensichtlich gehörte die Unterhaltung von Anfang an zu den wesentlichen Funktionen des Journalismus. Das legt nahe, den Journalismus nicht länger als Institution oder System zu betrachten, dessen vornehmste Aufgabe die politische Informationsvermittlung und -verarbeitung ist, sondern als Initiator des aktuellen gesellschaftlichen Zeitgespräches (vgl. Weischenberg 1992: 37ff.) und eines umfassenden kulturellen Verständigungsprozesses zu fokussieren.

Rühl hat mit seiner Definition von Journalismus als Herstellung und Bereitstellung von Themen zur öffentlichen Kommunikation (vgl. Rühl 1980: 319f.) die Basis für eine Entgrenzung des Journalismusbegriffes gelegt. Denn auch der Fernsehpfarrer Fliege stellt mit seiner Gesprächsrunde zweifellos Themen zur öffentlichen Kommunikation bereit und trägt so zur Selbstverständigung der Gesellschaft bei. Demgemäß haben Scholl (1997) und Weischenberg (1992: 47) den Journalismus ausdrücklich nicht nur auf seinen Informationsanteil begrenzt, sondern versucht, diesen durch das Merkmal Wirklichkeitsbezogenheit weiter zu fassen und zugestanden: „Unterhaltung kann durchaus Bestandteil des Journalismus sein, etwa in Form von Boulevardunterhaltung“ (Scholl 1997: 474). Das Zitat verdeutlicht jedoch auch, dass Unterhaltung nicht wirklich integriert wird, sondern das ungeliebte Wechselbalg bleibt, das den „eigentlichen“, seriösen Journalismus im Kern bedroht. 


\section{Probleme der empirischen Journalismusforschung}

Die auf die Aufklärungs- und Informationsfunktion beschränkte Sicht auf den Journalismus hat in der Journalismusforschung nachhaltige Spuren hinterlassen. Vor allem vier Problemfelder behindern unseres Erachtens ein angemessenes Herangehen und damit ein besseres Verständnis der gegenwärtigen Wandlungsprozesse:

- die Verengung der Journalismusforschung auf die Journalisten- und vereinzelt Journalistinnenforschung;

- die Fokussierung auf den Nachrichtenjournalisten in den vorliegenden Studien, die so lediglich Erkenntnisse über ein - zunehmend kleiner werdendes - Segment der Profession ermöglichen;

- damit einhergehend in der Analyse journalistischer Aussagenproduktion die Dominanz von Nachrichtensendungen und politischen Informationsprogrammen; schließlich:

- die zunächst mit der disziplinären Arbeitsteilung, im Weiteren aber auch theoretisch begründete Vernachlässigung des Publikums und des Rezeptionsprozesses in der Journalismusforschung.

\subsection{Journalismusforschung als Journalistenforschung}

Böckelmanns (1993) Synopse der zwischen 1945 und 1990 in der Bundesrepublik durchgeführten Kommunikatorforschung zeigt die überwältigende Dominanz akteurs- und berufsstandszentrierter Forschung, zu deren „Schlüsselkategorie“ in den 70er Jahren die journalistische Berufsrolle und das Rollenselbstbild werden (vgl. ebd.: 43). Das bis in die 80er Jahre hinein beklagte Wissensdefizit über Motive, Verhaltensweisen und Merkmale der journalistisch Handelnden haben vor allem die beiden Repräsentativerhebungen aus Münster und Hannover behoben (Scholl/Weischenberg 1998; Schneider u. a. 1993a, 1993b, 1994). Damit treten andere Lücken, jenseits der Akteurszentrierung, deutlicher hervor.

In der Folge des Paradigmenwechsels vom Subjekt zum System (vgl. Rühl 2000) wäre eine Abkehr von den KommunikatorInnen und eine Hinwendung zu den Strukturen der journalistischen Aussagenproduktion zu erwarten gewesen (vgl. dazu auch Scholl 1997). Theoretisch hat diese Wende mit der ausgiebigen systemtheoretischen Diskussion in den 90er Jahren auch stattgefunden, empirisch ist sie jedoch weitgehend ausgeblieben. Obschon Systeme ohne Subjekte modelliert werden und Kommunikation als Letztelement erkannt wird, bleibt der Kommunikator sowohl Ausgangs- als auch Drehund Angelpunkt der empirischen Journalismusforschung. Beispielsweise ist im Untersuchungsinstrument der Münsteraner Erhebung die Anlehnung an bereits vorliegende Journalistenbefragungen viel deutlicher als die Übereinstimmung mit den systemtheoretischen Prämissen. Die Kluft zwischen der grundsätzlichen Irrelevanz der Akteure in der Luhmann'schen Systemtheorie und ihrer praktischen Bedeutung für die strukturierte Befragung hat die systemtheoretisch fundierte Journalismusforschung noch nicht geschlossenen. Insbesondere sind die Leistungen, die der Journalismus im Blick der Systemtheorie für seine Umwelt erbringt, bislang kaum angemessen erfasst und beschrieben worden. Dafür müssten in jedem Fall andere methodische Zugänge gewählt werden als die Erhebung von Selbstbeschreibungen der Medienakteure auf Basis strukturierter Befragungsinstrumente.

Insgesamt erweist sich die deutsche Journalismusforschung in ihrer Konzentration auf die KommunikatorInnen als bemerkenswert resistent gegenüber handlungstheore- 
tischen und auch kulturwissenschaftlichen Ansätzen, die in den europäischen Nachbarstaaten seit Ende der 70er Jahre diskutiert und in den letzten Jahren weiter an Bedeutung gewonnen haben (vgl. Lünenborg 2000).

\subsection{Journalistenforschung als Forschung über politische Nachrichtenjournalisten}

Die deutsche Journalismusforschung beschränkt sich - teils ausdrücklich, teils unausgesprochen - zusätzlich auf den politischen Nachrichtenjournalisten. Ob in den früheren Kommunikatorstudien von Donsbach (1982), in denen er deutschen Journalisten eine übermäßige Meinungsfreude attestierte, oder in seinen neueren vergleichenden Arbeiten (Donsbach 1993, Patterson/Donsbach 1996) - stets basieren die Schlussfolgerungen auf Daten über Nachrichtenjournalisten. Sieverts (1998) Analyse des europäischen Journalismus legt diesen Journalistentypus genauso zugrunde wie Essers (1998) Vergleich deutscher und britischer Tageszeitungsredakteure. Aussagen über den Nachrichtenjournalisten scheinen der idealtypische Ausgangspunkt der Analysen zu sein, der Nachrichtenjournalist wird so zum Prototyp für den Journalisten schlechthin. Andere journalistische Tätigkeitsbereiche und weiter gehend auch andere Funktionsrollen, die mit der Ausdifferenzierung des Journalismus quantitativ und qualitativ an Bedeutung gewonnen haben, werden demgegenüber in der Journalistenforschung nur selten erwähnt. Die wenigen Studien, die andere journalistische Arbeitsbereiche untersucht haben, bestätigen auch deshalb diesen Befund, weil sie nicht den Anspruch erheben, Erkenntnisse über das gesamte journalistische Berufsfeld zu liefern, sondern die „redaktionellen Außenseiter" betrachten.

"Journalismus in Deutschland“ (vgl. Scholl/Weischenberg 1998) weicht in der Einbeziehung aller journalistischen Arbeitsbereiche auf den ersten Blick von dieser Regel ab. Zugleich entstand die Studie als Paralleluntersuchung zu Weaver und Wilhoits (1996) Untersuchung der „US News People“. Die Münsteraner Forschungsgruppe hat zwar versucht, die Fokussierung auf Nachrichtenredakteure und -journalistinnen zu lockern, dennoch scheint diese aber in der Untersuchungsanlage immer wieder durch und bestimmt wichtige Ergebnisse. In der Itembatterie, mit der das journalistische Selbstverständnis gemessen wird, beziehen sich 17 der insgesamt 21 Antwortvorgaben direkt oder mittelbar auf den Tätigkeitsbereich der aktuellen Informationsvermittlung. Damit wird implizit weiter vom Prototyp des Nachrichtenjournalisten ausgegangen. Lediglich die Kategorien „positive Ideale vermitteln“, „Lebenshilfe für das Publikum bieten“ sowie „dem Publikum Unterhaltung und Entspannung bieten“ weichen von diesem Typus ab und erfassen andere journalistische Tätigkeitsbereiche. ${ }^{3}$

\subsection{Die Begrenztheit der Nachrichtenforschung}

Jenseits der Journalistenforschung hat sich das Primat der Information in der Journalistik vor allem in einer Schwerpunktsetzung auf die Nachrichtenforschung nieder-

3 Scholl und Weischenberg (1998: 253) haben dieser von uns bereits früher geäußerten Kritik entgegengehalten, dass sich „mindestens die Hälfte (der Antwortvorgaben - d. V.) auch auf für andere Arbeitsrollen typische Kommunikationsabsichten" beziehen würden. Das Argument belegt jedoch eher den Vorwurf der Verzerrung als dass es ihn entkräftete, da lediglich etwa 18 Prozent der Befragten im Nachrichtenjournalismus tätig waren (vgl. Weischenberg u. a. 1994: 20). Kein anderer Journalistentypus, egal ob Sportjournalistin, Moderator oder Fachzeitschriftenredakteur, erhält auch nur annähernd die gleiche Relevanz. 
geschlagen. Dabei wird in der deutschen Forschung überwiegend von einem Informationsübermittlungsmodell ausgegangen: Nachrichten übermitteln Fakten an RezipientInnen. Ihre Qualität bemisst sich folglich am Wirkungsgrad dieser Informationen auf das Publikum. Dabei ergeben die vorliegenden Studien zur Nachrichtenrezeption einhellig, dass ZuschauerInnen Nachrichten nur sehr mangelhaft und unvollständig aufnehmen und verarbeiten (vgl. Merten 1985, Ruhrmann 1989). „Missverstehen“, „falsche Wiedergabe“ und „fehlendes Erinnern“ konstatiert die Wirkungsforschung (vgl. Ruhrmann 1994: 253 f.). Angesichts der neueren Entwicklungen in der Rezeptions- und Publikumsforschung erscheint die angenommene Forschungsperspektive wie auch die Interpretation der Ergebnisse gleichermaßen fragwürdig.

Für ihre Zuschauer seien Fernsehnachrichten vor allem Unterhaltungstexte, hielt Bogart bereits 1980 fest. ${ }^{4}$ Die Funktion von Nachrichten für die RezipientInnen besteht offenbar nicht vorrangig im Informationsgewinn oder Wissenserwerb, da die gelieferten Fakten häufig schon während des Rezeptionsprozesses vergessen werden. Produktionsintention und Rezeptionsinteresse scheinen sich, belegt ist das für den Bereich des Fernsehjournalismus, zu widersprechen, ohne dass diese Diskrepanz im Journalismus bewusst reflektiert worden wäre. Dahlgren (1988: 286) hat dazu bemerkt: „Interestingly enough, this does not seem to have any significant impact on TV journalists or producers. To the extent that one can detect changes in the audiovisual discourse (e. g. faster tempo) the trend seems to make informational comprehension all the more difficult. Yet public discussions about TV news are still premised on the informational model.“

Dahlgren vertritt die Position, dass das Informationsmodell der Nachrichten insgesamt wenig hilfreich ist, um diese als journalistische Form zu verstehen und schlägt stattdessen vor, Fernsehnachrichten als kulturellen Diskurs zu betrachten, mit einer im Vergleich zur informationellen Komponente stärker rituellen, symbolischen und mythischen Bedeutung. Die regelmäßige Form der Mediennutzung trägt dazu bei, den Tag zu strukturieren (vgl. Neverla 1992: 192 ff.). Tagesschau und Abendnachrichten markieren den Beginn des Feierabends, die Spätausgaben entlassen das Publikum in die Nachtruhe.

Hickethier (1997) hat mit seinem Entwurf einer Narrationstheorie der Nachricht auf die Bedeutung der „Erzählbarkeit“ von Informationen hingewiesen. Nachrichten können damit als moderne Erzählungen betrachtet werden, die dramaturgischen, stilistischen und sprachlichen Anforderungen moderner Mythen entsprechen müssen. „Telling a good story “ definiert dementsprechend Liebes (1994: 1) als zentrale journalistische Aufgabe. Nicht Informationsaneignung, sondern Zugehörigkeit zu einer Gesellschaft, deren Identität narrativ hergestellt wird, wäre dann die Funktion der Nachrichtenre-

4 Solche Erkenntnisse führten u.a. zur Unterscheidung von Informations- und Unterhaltungssuchern, zur Hervorhebung der Bedeutung von Gefühlen und Erregung in der Rezeption und zur Beobachtung der Wirkung von narrativen versus traditionellen Präsentationsstilen (vgl. Donohew u. a. 1988). Wie schwer das Genre Nachricht dem Publikum den Verstehensprozess macht, erläutert Lewis (1994). Er beschreibt die Nachricht mit ihrem Klimax-first-Aufbau als Anti-Narration, die der normalen Erzählstruktur entgegengesetzt ist. „One of the defining features of television news is the absence of narrative codes in its structure." (ebd.: 25) Insbesondere die Struktur und sprachliche Gestaltung der Nachricht ist es also, die das Verstehen und damit den Wissenserwerb erschwert. Lewis schlägt basierend auf seiner Analyse vor: „[T] $]$ he transformation of television news back into the form of historical narrrative would allow a greater public understanding of the links and determinations of historical processes." (ebd.: 40) 
zeption (vgl. dazu auch Koch 1990; Schmitz 1996; Teichert 1987). Die Widersprüche zwischen Rezeptionsverhalten und Produktionsintention können in den bisherigen theoretischen Konzepten der Journalistik kaum aufgelöst werden, weil beide dominierenden Paradigmen, „Subjekt“ und „System“, ein Informationsmodell zugrunde legen.

\subsection{Journalismus(forschung) obne Publikum}

Am Feld der Nachrichtenforschung zeigt sich exemplarisch ein allgemeineres Problem der Journalismusforschung in Deutschland: Diese hat im Zuge der - entlang der Laswell-Formel erfolgten - fachspezifischen Ausdifferenzierung die Auseinandersetzung mit dem Publikum an die Rezeptions- und Wirkungsforschung abgetreten. Als Folge dieser innerdisziplinären Arbeitsteilung hat die Journalistik wichtige theoretische Weiterentwicklungen und neue empirische Erkenntnisse in der Publikumsforschung kaum zur Kenntnis genommen. So basieren die normativ aufgeladenen Dualismen von „Seriösem und Boulevardeskem“, „Information und Unterhaltung“, „Aufklärung und Schreinemakerisierung“, dem „Wichtigem und dem Populären“ auf einem Journalismusbegriff, der ohne das Publikum auskommt.

Die Einführung der Publikumsperspektive in die Journalismusforschung, wie sie Weiss bereits 1977 (in Böckelmann 1993: 21) vorgeschlagen hat, ist u. a. mit der Begründung abgelehnt worden, dass Kommunikatorforschung dann ausufern und zur Kommunikationsforschung schlechthin würde. Das kann deshalb nicht befriedigen, weil das Publikum implizit immer Maßstab zur Beurteilung der Medienakteure und ihrer Produkte war. Besonders deutlich tritt das in den Arbeiten von Noelle-Neumann, Kepplinger und Donsbach zutage (zusammenfassend Böckelmann 1993: 23; vgl. auch Donsbach 1999), deren Ansatz ein Publikum voraussetzt, das die Medieninhalte überwiegend passiv konsumiert und den Einflüssen der Journalisten recht hilflos ausgesetzt ist. Damit wurde eine Sicht auf das Publikum beibehalten, die in der Publikumsforschung heute als überholt gilt. Hier wird fast ausnahmslos die Annahme eines „aktiven Publikums“ akzeptiert (vgl. Hasebrink/Krotz 1996). Die mangelnde Reflektion dieser veränderten Sichtweise auf das Publikum führt in der Journalistik vor allem deshalb zu Problemen, weil die sich derzeit im Journalismus vollziehenden Veränderungen maßgeblich mit einer verstärkten Publikumsorientierung zusammenhängen.

Die Hinwendung zur Systemtheorie hat den weitgehenden Ausschluss des Publikums aus der Journalismusforschung nicht etwa korrigiert, sondern scheinbar noch theoretisch abgesichert. „(...) Luhmann benötigt die Inklusion des Publikums für seine Beschreibung des Systems Massenmedien nicht, weil mit der Produktion massenmedialer Inhalte die Systemoperationen abgeschlossen sind. Die Rezeption des Publikums ist Nebensache und nicht mehr konstitutiv für die Systemprozesse." (Scholl/Weischenberg 1998: 120, Fn. 18) Nicht alle Vertreter einer systemtheoretisch argumentierenden Journalismusforschung stimmen dem jedoch zu. Marcinkowski (1993: 78) erkennt das Publikum als „innere Umwelt des Journalismus“. Blöbaum (1994: 167) beschreibt das Publikum als Bestandteil des Systems in der Komplementärrolle zur Handlungsrolle der Journalisten. Rühl (1993: 142) erweitert seine eigene systemtheoretische Perspektive der Massenmedien in einer medienökonomischen Version, die das Publikum als „unorganisierte Annehmer" und „Verarbeiter" als systemzugehörig erkennt, damit aber das System Massenmedien als Teilsystem der Ökonomie unspezifisch werden lässt.

Diese Versuche, das Publikum in das System Journalismus zu integrieren, blieben jedoch empirisch folgenlos und erscheinen auch theoretisch problematisch. Die Systemtheorie setzt voraus, dass System und Umwelt durch einen binären Code eindeutig von- 
einander abgegrenzt werden können und auf dieser Basis auch die Zugehörigkeit oder Nichtzugehörigkeit zum System jederzeit eindeutig bestimmt werden kann. Die Publikumsrolle im System Journalismus ist jedoch gerade durch ihre Unspezifik gekennzeichnet. Nur die tatsächlichen Publika spezifischer journalistischer Angebote, nicht aber das potenzielle, disperse Publikum der Massenmedien ist von der Gesamtgesellschaft zu unterscheiden, weil sich in der Mediengesellschaft niemand dem Einfluss der Medien vollständig entziehen kann. Wird das Publikum als zum System zugehörig erkannt, so folgt daraus, dass eine Grenzziehung zwischen System und Umwelt nicht mehr möglich ist und der Systembegriff damit seine primäre Unterscheidungsfunktion verliert.

Die Verschlossenheit der aktuellen deutschen Journalismusforschung gegenüber einer Integration der Zuschauerperspektive bleibt erstaunlich, weil mit dem Einzug des (radikalen) Konstruktivismus in die Journalistik von einem veralteten Objektivitätsdenken Abschied genommen wurde (vgl. Merten u. a. 1994). Die Prämissen des Konstruktivismus, nach denen Gesellschaft und Kultur von den sozialen Subjekten kommunikativ hergestellt werden und deshalb immer subjektabhängig sind, haben aber nur auf Seiten der Kommunikatoren und Aussagenproduzentinnen, nicht auf Seiten des Publikums in die Theoriebildung Eingang gefunden. ${ }^{5}$ Wenn die Sinnkonstitution subjektabhängig erfolgt, dann gilt das gleichermaßen für alle Medienhandelnden, egal ob sie als Journalisten oder als Publikum in den Prozess der gesellschaftlichen Bedeutungsproduktion durch Medientexte einbezogen sind. Texte vermitteln deshalb keine fixierte Bedeutung, machen „für sich“ keinen Sinn, vielmehr erhalten sie Sinn und Bedeutung durch die interpretativen Aktivitäten der sozialen Subjekte, die in den Diskurs über Medienangebote einbezogen sind. Deshalb kann nur eine Journalismusforschung, die die Publikumsperspektive integrativ in ihre Forschungstätigkeit mit einbezieht, Aussagen über die gesellschaftliche Relevanz journalistischer Textangebote treffen.

\section{Journalismus unter der Perspektive der Cultural Studies}

Die Cultural Studies, die in der deutschsprachigen Rezeptionsforschung zunehmend an Bedeutung gewinnen, stellen einen geeigneten theoretischen Rahmen bereit, um die derzeitigen Wandlungsprozesse im Journalismus analytisch zu erfassen. Sie enthalten damit das Potenzial, einen theoretischen Zugang zur empirischen Journalismusforschung zu finden, der ihre beschriebene Engführung erweitert. Die folgenden Ausführungen verstehen wir als erste Überlegungen dazu, wie eine solche handlungs- und kulturorientierte Journalismusforschung konzipiert werden kann.

5 Eine partielle Ausnahme bildet die von Schmidt und Weischenberg (1994) vorgelegte Auseinandersetzung mit Mediengattungen. Hier wird der Gattungsbegriff nicht ontologisch gefasst, sondern unter Bezugnahme auf das Handeln des Publikums entwickelt: „Erfolgversprechender scheint jedoch zu sein, das Verhalten von Menschen im Umgang mit Medien daraufhin zu untersuchen, wie sie mit Gattungsbezeichnungen umgehen und welches Wissen sie mit solchen Bezeichnungen verbinden." (ebd.: 212) Auffällig ist jedoch, dass diese konstruktivistisch fundierte Erkenntnis von Mediengattungen bei der im selben Artikel erfolgenden Beschäftigung mit journalistischen Darstellungsformen keine Anwendung mehr findet. 


\subsection{Grundlagen der Cultural Studies}

In den Cultural Studies ${ }^{6}$ steht das handelnde Subjekt in seiner sozialen und historischen Kontextuierung im Mittelpunkt der Auseinandersetzung. Im Vergleich zur Systemtheorie bedeutet diese theoretische Prämisse keinen „Rückfall“ zu einem subjektzentrierten Paradigma, das Gesellschaft auf die Ansammlung von Individuen reduziert. Die handlungs- und kulturtheoretische Orientierung der Cultural Studies ermöglicht vielmehr, „die Erforschung der unterschiedlichen Manifestationen des kulturellen Lebens und Handelns in Alltagskontexten" (Göttlich/Winter 1999: 29). Das kulturell gebundene Handeln der Subjekte in ihren sozialen Kontexten soll beschrieben und als Prozess der Positionierung und Selbstpositionierung analysiert werden. Die Frage nach Macht und deren Verteilung innerhalb der Gesellschaft rückt damit ins Zentrum der Forschung - ein zentraler Unterschied zur Systemtheorie, die Dominanzverhältnisse und Interessenkonflikte bzw. die daraus resultierenden Differenzen grundsätzlich als sekundär betrachtet.

Cultural Studies als theoretischen Zugang zur Medienwissenschaft zu nutzen, impliziert eine veränderte Sicht auf Medien, denen im Dreieck mit Kultur und Macht eine zentrale gesellschaftliche Rolle zukommt. Dabei werden Medientexte in ihrer Eigenschaft gesehen, Material für die (hegemoniale) Bedeutungsproduktion zu liefern und zugleich Mittel zur Verhandlung und Neuorganisation gesellschaftlicher Dominanzverhältnisse bereitzustellen. Das ist möglich, weil Medien lebensweltlich verortet werden, als Bestandteil der Alltagskultur und der sich im Alltag entfaltenden Lebensweisen. Diese andere Sicht auf Medien und ihre gesellschaftliche Funktion, wie sie seit Mitte der 70er Jahre in England und den USA herausgearbeitet wurde, eröffnete neue Forschungsperspektiven: Zum einen fanden bei den Cultural Studies von Anfang an Formen der Populärkultur in besonderem Maße Aufmerksamkeit. Im Bereich der fiktionalen Medienangebote wurden vor allem Serien und dabei insbesondere das Soap-Opera-Genre untersucht (vgl. für einen Überblick Klaus 1998: 321 - 373). Zum anderen wandten sich die Cultural Studies als Erste unter den kritischen Medientheorien konsequent dem Rezeptionsprozess und dem Publikum als Instanz der Bedeutungsproduktion zu.

„Popular culture is not consumption, it is culture - the active process of generation and circulation of meanings and pleasures within a social system: culture, however industrialized, can never be adequately described in terms of buying and selling of commodities." (Fiske 1989: 23) Aufgrund ihrer Offenheit liegt in dieser Sichtweise auf das Publikum aber auch die Gefahr der Beliebigkeit und der Verkennung des Machtgefälles zwischen Medienproduktion und Rezeption (vgl. Bromley 1999: 21; Ferguson/Golding 1997). Selbst Fiske (1989), dem dieser Vorwurf häufig gilt, betont jedoch, dass die dem Text innewohnende Offenheit, seine polyseme Struktur, niemals vollständig ist, sondern bestimmte Deutungen vor anderen präferiert (vgl. Winter 1997). Der Blick auf das Publikum im Rahmen der Cultural Studies - und im Unterschied zum Uses-and-Gratifications-Approach - muss deshalb nach Morley (1996: 41) ein Blick auf „Interaktionen in ihrem sozialen Kontext“ sein: „Soziales Handeln (und als solches wird Medienrezep-

6 Die Textbücher von Hepp (1999), Hepp/Winter (1997) sowie Bromley/Göttlich/Winter (1999) führen in die Entstehungsgeschichte und die Grundlagen der Cultural Studies in Großbritannien, den USA und Australien sowie ihre Rezeption in Deutschland ein. Einen Überblick über die kritische Auseinandersetzung mit den aktuellen Entwicklungen bieten Ferguson/Golding (1997). 
tion hier handlungstheoretisch betrachtet - d. V.) ist immer auf seine ,Umgebung', auf seinen Kontext gerichtet, und Umgebung oder Kontext sind nichts Sekundäres oder bloß externer Hintergrund von Handeln."

Die theoretischen Konzepte, die der Neubeschreibung von Publikumsaktivitäten im Rahmen der Cultural Studies zugrunde liegen - Ideologie, Hegemonie und Polysemie sind allesamt auch für die Seite des journalistischen Handlungszusammenhanges relevant. In Halls (1973) ursprünglichem Kodierungs-/Dekodierungsmodell sind die verschiedenen Seiten der Medienproduktion und des Journalismus auch integrativ zusammengedacht. Die Prozesse der Kodierung wie auch der Dekodierung werden nach Hall vor allem durch drei Bezugsgrößen bestimmt: „frameworks of knowledge, relations of production, technical infrastructure“. Johnson (1985) hat diesen Gedanken in seinem zirkulären Modell, in dem Produktion, Text, Lesweisen und „gelebte Kulturen“ miteinander verbunden sind, weiter ausgeführt.7 Selbst wenn in empirischen Untersuchungen zumeist eine Konzentration auf einen der zentralen Aspekte des skizzierten „Kulturkreislaufes“ erfolgt, müssen nach Johnson die anderen jeweils in der Konzeption und Durchführung der Forschung mit berücksichtigt werden. Zoonen (1994: 8) hat dazu ausgeführt: „A crucial feature of the encoding/decoding model is that media discourse is supposed to be produced by media institutions and audiences at the same time, not as an activity of single institutions or individuals but as a social process embedded in existing power and discursive formations."

Diese Herausforderung ist bislang nur unzureichend eingelöst. Forschungsprojekte in der Tradition der Cultural Studies haben sich überwiegend auf die Publikumsforschung beschränkt und damit die Möglichkeit, Produktions- wie Rezeptionsprozesse ausgehend von denselben theoretischen Prämissen zu analysieren, noch nicht ausgeschöpft. Die nahezu vollständige Konzentration auf Rezeptionsanalysen und damit verbunden eine „Überbewertung der Semiologie und des Textes als vorrangigem (wenn nicht gar einzigem) Ort der Bedeutung und Bezeichnung" ist im Rahmen der derzeit zu beobachtenden kritischen Bestandsaufnahme der Cultural Studies vermerkt worden (Bromley 1999: 21). Entsprechend fordert auch Hepp (1999: 262) „nicht mehr nur den Bereich der Medienaneignung zu fokussieren ..., sondern auch unterschiedliche Produktionskulturen." Mit der vorrangigen Rezeptionsorientierung ergibt sich zugleich die Gefahr einer theoretischen Verkürzung durch die Nicht-Unterscheidung von Produzent und Text in der Analyse. Jäckel und Peter (1997) haben auf das Problem aufmerksam gemacht, dass der Diskurstheorie zufolge ein Text erst in dem Augenblick konstituiert wird, in dem RezipientInnen ihn mit spezifischen Bedeutungen belegen - Fiskes „reader-as-writer“ Position. Während damit die Dekodierung als eigenständige Leistung der RezipientInnen erkannt ist, verschwindet der Kodierungsprozess. Zahlreiche Studien gehen davon aus, dass die Bedeutungszuweisung im Kodierungsprozess dem Text eindeutig zu entnehmen sei. Dieser Produktionsprozess ist jedoch zunächst einmal als genauso wenig transparent wie der Rezeptionsprozess anzunehmen.

Im Kontext der kulturorientierten Publikumsforschung haben sich Studien in der Tradition des „popular culture projects“ (Corner 1991) auf das alltägliche Vergnügen der Zuschauer bei der Rezeption von vorwiegend fiktionalen Sendungen beschränkt. Wenn journalistische Aussagenproduktion im Rahmen der „Audience Studies“ überhaupt betrachtet wurden, dann vor allem im Hinblick auf das „public knowledge pro-

7 Bechdolf (1999) hat das Modell zur Grundlage ihrer Rezeptionsstudie gemacht und damit an seine empirische Relevanz erinnert. 
ject“. Theoretisch stand in dessen Mittelpunkt eine Auseinandersetzung mit Ideologie und der Durchsetzung hegemonialer Bedeutungen (vgl. Hall 1989), empirisch beruhte es fast immer auf einer Analyse der Rezeption von Nachrichtensendungen (vgl. Jensen 1988; Dahlgren 1988; Morley 1980).

Hall (1973) hat dazu mit seinem Vorschlag, drei Lesarten der Rezeption zu unterscheiden - die dominante, die ausgehandelte und die oppositionelle -, den Grundstein gelegt. Sein Modell konnte unmittelbare Plausibilität vor allem für politische Informationssendungen beanspruchen, weil deren ideologische Funktion zur Absicherung der ökonomischen und politischen Dominanzverhältnisse explizit hervortritt. Halls Modell ist inzwischen mehrfach revidiert und erweitert worden. So hat Dahlgren (1988) unterschiedliche Formen des Sprechens über Fernsehnachrichten beschrieben. Er unterscheidet dabei auf der ersten Ebene zwischen dem official discourse und dem personal discourse. Weiter gehend unterscheidet er in den beiden Diskurstypen differente Formen des Sprechens über Fernsehnachrichten im Alltag (vgl. ebd.: 292 ff.). Begründet in der Offenheit des Textes, sind vielfältige Deutungen in Abhängigkeit von den Rezeptionskontexten möglich. Dahlgren (1988) zufolge ist Bedeutungszuweisung in der Rezeption vielfältig, sozial gebunden, in Subkulturen verortet und kontinuierlich fortlaufend. ZuschauerInnen „lesen“ den angebotenen Fernsehtext der Nachrichten also unterschiedlich in Abhängigkeit von ihrer sozialen Position und der Eingebundenheit in spezifische Kontexte. Bedeutung gewinnen Nachrichten keineswegs direkt zum Zeitpunkt des Sehens, sie konstruiert sich vielmehr fortlaufend: „Meaning, in other words, never achieves final stability though the processes themselves may well take on the quality of the routine“ (Dahlgren 1988: 290 f.). Die Schlussfolgerungen aus diesen Studien sind für den aktuellen Nachrichtenjournalismus vernichtend: „The audiovisual discourse of TV news occludes social reality precisely in making available an array of possible meanings, none of which on their own terms invite or help the viewer to locate him/herself as a political subject in an historical setting " (ebd.: 299 f.). Dahlgren fordert demzufolge, dass die wissenschaftliche Auseinandersetzung mit dem Fernsehproduktionsprozess diese Erkenntnisse der Rezeptionsforschung mit einbezieht. Eine Forderung, die bis heute uneingelöst blieb und in der deutschen Journalismusforschung aufgrund ihrer programmatischen Exklusion des Publikums bislang nicht umsetzbar ist.

Zusammenfassend halten wir fest: Wenn sich auch bislang die empirische Forschung auf den Rezeptionsprozess konzentriert hat, so ist das Konzept der Cultural Studies keineswegs darauf beschränkt. Eine kulturtheoretisch fundierte und somit „radikal kontextuierte“ (Ang) Journalismusforschung umfasst vielmehr eine Beobachtung und Analyse des Produktionsprozesses, des Produkts und des Rezeptionsprozesses in ihrer jeweiligen kulturellen Eingebundenheit. Sie analysiert wie gesellschaftliche Macht und Bedeutung in journalistische Texte eingeschrieben wird, wie sie sich im Text semiotisch darstellt und in welcher Weise diese Bedeutung vom Publikum im Alltag aktiv angeeignet wird und dann auf den Produktionsprozess zurückwirkt. Journalismusforschung beobachtet, wie im Prozess der gesellschaftlichen Selbstverständigung, den der Journalismus federführend gestaltet, gemeinsamer kultureller Sinn durch das Aushandeln von Bedeutungen produziert wird. Von besonderer Relevanz sind dabei die Widersprüche und Konflikte, die zwischen den medialen Bedeutungsangeboten und der kontextgebundenen Bedeutungsproduktion des Publikums sichtbar werden. 


\subsection{Cultural Studies in der Journalismusforschung - ein Überblick}

Hat der Cultural Studies Ansatz in Deutschland insgesamt mit deutlicher Verspätung und am Rande der Medienwissenschaft Einzug gehalten, so hat er die Journalismusforschung bis heute nicht erreicht. Mit einer Ausnahme (vgl. Renger 1997, 2000) liegt in der deutschsprachigen Journalismusforschung bislang keine kulturtheoretische Auseinandersetzung mit dem gesellschaftlichen Prozess der Bedeutungsproduktion von Journalismus als Zusammenwirken von Kommunikatoren und RezipientInnen vor. Renger (2000) zeigt in seiner historischen Annäherung die Bedeutung populärkultureller journalistischer Produkte für die Entwicklung des Mediensystems detailliert auf. Die Cultural Studies, so argumentiert er überzeugend, ermöglichen, die Erscheinungsformen der „popular culture“ in den Medien zu verstehen und ihre Entwicklung nachzuvollziehen. Damit schließt Renger an Diskussionen im englischsprachigen Raum an, die den Cultural Studies ein besonderes Erklärungspotenzial für den Boulevardjournalismus attestieren (vgl. Dahlgren 1992; Sparks 1992). Diese Perspektive bleibt jedoch problematisch: Mit der Behandlung der bisher ausgegrenzten journalistischen Formen wird zwar eine wichtige Forschungslücke geschlossen, zugleich bleibt aber der Dualismus von informations- versus unterhaltungsorientierten Produktionen intakt. Ungewollt reproduzieren die Autoren den Antagonismus zwischen „popular culture“ und „public knowledge“. Damit aber wird jenes Integrationspotenzial der Cultural Studies nicht genutzt, das die Trennung zwischen dem Informationsangebot als traditionellem Kern des Journalismus und dem Unterhaltungsangebot als seinem boulevardesken Randbereich überwindbar werden lässt.

Auch Langer (1998) nimmt in seiner Untersuchung der „anderen“ Nachrichten darunter fasst er die als banal bewerteten Meldungen über Katastrophen, Verbrechen und tragische Schicksale - die Trennlinie zu den "seriösen“ Nachrichten zum Ausgangspunkt. Insbesondere auf dem Feld der „other news“ könnten widersprüchliche Tendenzen der TV-Nachrichtenproduktion an die Oberfläche gelangen, weil diese als Texte offener sind und einer weniger starken hegemonialen Kontrolle unterliegen. Langers Ergebnisse weisen jedoch über den zugrunde gelegten Dualismus hinaus, wenn er festhält, dass die Boulevardnachrichten die gleichen ideologischen Muster anbieten wie die „seriösen“ Nachrichten. „The ,other news' offer modes of explanation and sense-making which displace and mask the social, political and historical context in which events occur and can be made to mean." (ebd.: 150) Er arbeitet in seiner Analyse anhand der Personendarstellung in den „other news“ zwei solcher Erzählweisen heraus, die gleichermaßen soziale Ungleichheit verdecken und zur Stabilisierung der gesellschaftlichen Machtverhältnisse führen. Die erste Erzählung kreist um die „kleinen Leute“, die durch außergewöhnliche Leistungen jederzeit aus der Masse hervortreten und damit zu einer prominenten Persönlichkeit werden könnten. Die zweite Erzählung in den „anderen Nachrichten“ handelt demgegenüber von den gewöhnlichen Taten, dem unspektakulären Verhalten der Mächtigen, Großen und Reichen, die so zu „einem von uns“, einem Gesellschaftsmitglied unter anderen würden. Langers Befunde stützen empirisch Dahlgrens (1992) Vorschlag, von einem Kontinuum des Geschichtenerzählens auszugehen. In dieses Kontinuum sind „serious“ und „tabloid“ Nachrichten, Fakt und Fiktion, Journalismus und Populärkultur als verschiedene, komplementär wirkende Dimensionen eingebunden. Die Journalismusforschung kann demnach nicht länger von zwei gegensätzlichen Arten medialer Produktion ausgehen. Sie muss vielmehr graduelle Unterschiede der verschiedenen Medientexte herausarbeiten, die sich in Stil, Form, Genrezugehörigkeit, Erzählweise, Faktendichte, Übermittlungs- 
leistung, Publikumsansprache, ideologischer Aussage und diskursiven Anschlussmöglichkeiten zeigen.

Hartley (1996) hat versucht, in seiner Konzeptionierung des Journalismus als „the textual system of modernity“ die binären Zuschreibungen aufzulösen. Seine Bearbeitung des Zusammenhanges von Journalismus, Moderne und Populärkultur konzentriert sich jedoch ausschließlich auf die Rezeptionsseite. Er ignoriert den Produktions- und Herstellungsprozess von Texten und fixiert gesellschaftliche Bedeutung lediglich auf Seiten der Publika bzw. der Öffentlichkeiten. Das gilt für die Mehrzahl der Arbeiten, die sich im Rahmen der Cultural Studies mit journalistischen Leistungen beschäftigt haben. So hat Jensen (1988) anhand dänischer Fernsehnachrichten die Formen der Aneignung durch das Publikum herausgearbeitet, die die Welt der Nachrichten und die Welt des Alltags verbinden. Er strukturiert diese Deutungsleistungen des Publikums mittels „Superthemen" wie Krieg, Umwelt und Arbeitslosigkeit. Solche Themen stellen Jensen zufolge das Bedeutungspotenzial bereit, das in Gesprächen über die Nachrichteninhalte aktiviert werden kann und erst in dieser Form des sekundären Medientextes sichtbar wird. Die oben bereits erwähnten Arbeiten von Dahlgren $(1992,1988)$ knüpfen an Jensens Studie an und vertiefen die unterschiedlichen Formen der Aneignung und Bedeutungszuweisung von Fernsehnachrichten. Im Mittelpunkt dieser und anderer Studien zur Nachrichtenrezeption steht die Erkenntnis, dass dieselben Medientexte in Abhängigkeit vom sozialen, kulturellen und historischen Kontext sehr unterschiedliche Deutungen erfahren. Erst wenn überhaupt ein Bezug zum eigenen Lebenskontext hergestellt werden kann, setzt der Prozess des Verstehens ein. Fiske (1989) hat genau dies zum Kriterium „populärer Texte“ erklärt, die ihren RezipientInnen erlauben, hegemoniale Bedeutungen zu unterwandern.

Sparks (1992) nutzt den Cultural Studies Ansatz für seine theoretische Auseinandersetzung mit den Texten der britischen tabloids. Er systematisiert die britische Presse in popular, middle und quality press und hält damit die Unterscheidung zwischen dem „popular culture project" und dem "public knowledge project“ aufrecht. Dabei vermag Sparks in den tabloids nicht das kritische Potenzial zu entdecken, das Fiske und andere der Populärkultur zusprechen: „In order to argue that the popular can be the site of some sort of liberating political practice it would be necessary to show how it is that the concerns of the everyday may be used to construct a more generalized oppositional position which is capable of transcending the limits of orthodox political power and providing the intellectual material for self-liberation" (Sparks 1992: 42). Ähnlich argumentieren auch Bruck und Stocker (1996), die „die ganz normale Vielfältigkeit des Lesens“ in ihrer Rezeptionstudie der österreichischen Neuen Kronen Zeitung (NKZ) herausstreichen. „Wenn Boulevardmedien nach dem Prinzip Vorurteil statt Kritik und Gefühl anstelle von Intelligenz funktionieren, dann ist auch das Bild von den sich informierenden LeserInnen zu korrigieren. Die Palette der von uns beobachteten Leserollen reicht von Spektakel und Aufregung suchenden VoyeurInnen bis zum Fußballer, der jedes statistische Detail im Sportteil genau studiert." (ebd: 295) Die Autoren betonen die lebensweltliche Einbindung des Rezeptionsprozesses, den die kritische Medienforschung zur Kenntnis nehmen müsse, um die gesellschaftliche Relevanz von Populärmedien einschätzen zu können.

Journalismus basiert in der Perspektive der Cultural Studies nicht länger auf dem Dualismus von Information und Unterhaltung, von Seriösem und Boulevardeskem, von qualitativ Hochwertigem und Trivialem. Stattdessen ermöglicht die Kulturorientierung, Journalismus als Form der diskursiven Auseinandersetzung mit der Wirklichkeit zu betrachten, an der Kommunikatorinnen wie Rezipienten gleichermaßen beteiligt sind. 
Hermes (1997) hat sich mit der Frage beschäftigt, wie in der Rezeptionsforschung der Dualismus von Unterhaltung und Information, Vergnügen und Politik konzeptionell überwunden werden könnte. Sie plädiert für das Konzept der „cultural citizenship “ und argumentiert, dass dieses Konzept funktionieren könnte als „a crowbar to pry apart practices and identities, or as a means to mix in issues of pleasure with issues of politics, it could help redefine the boundaries of the public and the private in a firm insistence on how both are articulated on the level of the everyday and are reciprocally involved in how we constitute ourselves in relation to society. " (Hermes 1997: 88)

Im Rahmen der hier diskutierten Fragestellung verdient dieser Vorschlag deshalb Aufmerksamkeit, weil das Konzept ursprünglich von Allor und Gagnon (1994) stammt, die mit der Einführung der „cultural citizenship“ eine theoretische Verbindung zwischen dem Produktions- und dem Konsumtionsbereich der Medien herstellen wollten. Indem sie die politische Kultur als diskursiv hergestellt auffassen, verweisen sie auf die Notwendigkeit, die unterschiedlichen diskursiven Praktiken, die im Produktions- und im Rezeptionsprozess, im journalistischen Herstellungsprozess und im alltäglichen Aneignungsprozess der Massenmedien wirken, zu untersuchen.

Die für die Rezeptionsforschung produktive Feststellung der grundsätzlich polysemen Struktur von Medientexten hat aber nicht nur Konsequenzen für den Dekodierungsprozess, also die Lesarten der Texte. Sie muss gleichermaßen auch im journalistischen Produktionsprozess Wirkung entfalten und wirft dort die Frage nach Formen der Kodierung von Bedeutung auf. Dieser Prozess ist bisher viel seltener untersucht worden. Jedoch kann parallel zur Diskussion um die Lesarten von Texten davon ausgegangen werden, dass Journalistinnen und Journalisten in einem spannungs- und konfliktgeladenen Produktionsprozess spezifisch positioniert sind. Diese Positionierung erfolgt auf zwei miteinander verwobenen Ebenen: zum einen den ressort- und medienspezifischen Anforderungen der Berufsrolle und zum anderen den subjektabhängigen und milieuspezifischen Dispositionen der journalistisch Handelnden. So entsteht ein Handlungsraum, in dem Medientexte kein geschlossenes Bedeutungssystem vorgeben, sondern Widersprüche und Konflikte reflektieren. Sie entstehen beispielsweise zwischen journalistischen Qualitätsansprüchen und organisatorischen, ökonomischen oder technischen Vorgaben, zwischen Redaktionsroutine und kreativem Berufsbild, zwischen externer Wertschätzung aufgrund des Medien- und Berufsimages und interner Geringschätzung innerhalb der redaktionellen Hierarchie, zwischen geringer gesellschaftlicher Bedeutung einzelner Ressorts oder Medien und hoher Berufsidentifikation oder auch zwischen bewusster politischer Haltung und unbewusster gesellschaftlicher Bedeutungsproduktion. Die aus solchen Widersprüchen resultierende potenzielle Vielfalt, die „semiotische Demokratie“ der individuellen journalistischen Bedeutungsproduktion, wird dadurch gebändigt, dass im Rahmen der bestehenden kulturellen Übereinkünfte manche Deutungen vor anderen favorisiert werden. Dies setzt allerdings keineswegs stets einen bewussten Entscheidungsprozess auf Seiten der Kommunikatoren und Textproduzentinnen voraus, denn die journalistische Konstruktion von Bedeutung ist in der Regel strukturell in die bestehenden Machtverhältnisse eingebunden. Zu vermuten ist deshalb vielmehr, dass widersprüchlich verlaufende Textproduktion nur dort tatsächlich bewusst reflektiert wird, wo sie verbreiteten gesellschaftlichen Deutungsmustern und hegemonialen Zuschreibungen entgegenläuft.

Zusammenfassend können wir festhalten, dass die Cultural Studies den Journalismus als einen wesentlichen Bereich gesellschaftlicher Bedeutungsproduktion und -zirkulation ansehen. Auch wenn mit Blick auf die Journalismusforschung gilt, dass die vor allen Dingen im englischsprachigen Raum vorgelegten empirischen Untersuchungen das 
theoretische Potenzial der Cultural Studies nicht ausgeschöpft haben, so verweisen sie doch auf die Notwendigkeit einer erweiterten Perspektive der Journalistik:

- Unterhaltung und Unterhaltungsangebote sind für den Journalismus konstitutiv. Die aus der Aufklärung stammende Sicht auf den Journalismus als Informationsjournalismus und den Nachrichtenjournalisten als Prototyp der Medienakteure muss dann überwunden werden.

- Als „textual system of modernity“ organisiert der Journalismus einen gesellschaftlichen Selbstverständigungsprozess. Die gesellschaftliche Bedeutung dieser Selbstverständigung kann nur durch eine Integration des Rezeptionsprozesses in die Journalismusforschung erfasst werden. Die nahezu ausschließliche Konzentration auf die Produzenten der journalistischen Texte verunmöglicht eine Analyse der konflikthaften Aushandlung von Hegemonie und Macht. Sie setzt die (zumeist hegemoniale Bedeutung) der Medientexte in eins mit der (unter Umständen abweichenden) Dekodierung durch das Publikum.

- Nachrichten - das zentrale Genre des Informationsjournalismus - gewinnen in der Perspektive der Cultural Studies eine veränderte Bedeutung. Sie gelten weniger als Form der Faktenvermittlung, denn vielmehr als rituelle Form der Vergewisserung über das Selbst im kulturellen Kontext. Als Genre unter anderen sind Nachrichten auf dem Kontinuum des journalistischen Geschichtenerzählens platziert, das insgesamt eine Teilnahme an der „Cultural Citizenship“ erlaubt.

\section{Der Kreislauf medialer Produktion und Rezeption}

Abschließend wollen wir ein Forschungsprogramm vorschlagen, das eine Perspektive eröffnet, die die Journalismusforschung entgrenzen und ihre beschriebenen Lücken und Defizite überwinden könnte. Dabei knüpfen wir an den bereits oben erwähnten „Kreislauf der Produktion und Konsumtion kultureller Produkte“ von Johnson (1985) an, den wir als Kreislauf medialer Produkte für eine kulturorientierte Journalismusforschung nutzen wollen.

Johnson unterscheidet vier Haupterscheinungsbilder von Kulturkreisläufen: 1. die Produktion und ihre Bedingungen, 2. Texte bzw. Formen, 3. Lesweisen und ihre Bedingungen und 4. gelebte Kulturen, die sich mit Sozialbeziehungen kreuzen. Wir nutzen das Modell zur Darstellung des Kreislaufs der medialen Bedeutungsproduktion (vgl. Abb). Dabei stellen gelebte Kulturen oder Milieus für Johnson das Bindeglied zwischen privaten und öffentlichen Lebensäußerungen und die Schnittstelle zwischen kultureller - hier: massenmedialer - Produktion und ihrer Konsumtion dar. „Alle Momente oder Aspekte hängen voneinander $\mathrm{ab}$ und sind für das Ganze unverzichtbar. Jedes ist aber auch ein besonderes, und es bringt charakteristische Formveränderungen mit sich. Daraus folgt, dass wir, wenn wir an einem Punkt des Kreises stehen, nicht unbedingt sehen, was an anderen geschieht." (ebd.: 25) In Anlehnung an Hermes (1997) verwenden wir den Begriff der „Cultural Citizenship“ anstelle der „gelebten Kulturen“, um die gesellschaftliche Dimension der Bedeutungskonstruktion zu beschreiben.

Johnson plädiert nun nicht dafür, immer alle Momente des Kreislaufes gleichermaßen zu betrachten, weil er darin eine Überforderung von empirischer Forschung sieht, die zu unzulässigem Reduktionismus führen könnte. Gleichzeitig arbeitet er aber heraus, dass aus der Ignorierung des Zusammenspiels der verschiedenen Momente des Kulturkreislaufes eine - von uns auch für die Journalistik und Kommunikationswissenschaft beobachtete - theoretische und disziplinäre Fragmentierung erwächst, die zu Fehlinterpretationen, blinden Flecken und Erkenntnisgrenzen führt. 
Abb.: Zirkel der Bedeutungsproduktion angelebnt an Jobnson (1985: 24)

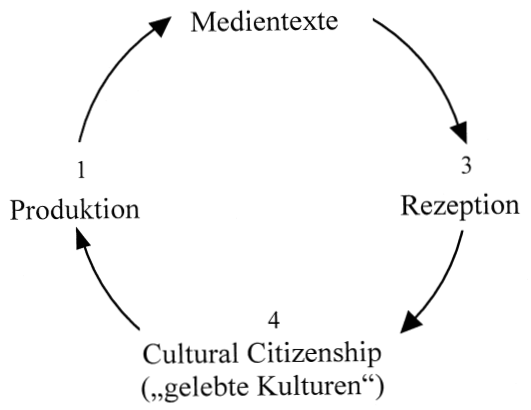

Nach Johnson weisen die vorliegenden produktionsorientierten Ansätze vor allem zwei Begrenzungen auf:

- Der „Ökonomismus“ berücksichtigt nicht die duale Natur des Kreislaufes kultureller Waren, der neben den ökonomischen Bedingungen auch durch den Vorrat bereits existierender kultureller Bedeutungen und zirkulierender Diskurse bestimmt wird.

- Der „Produktivismus“, der den Charakter eines Kulturproduktes und seiner sozialen Folgen aus den Bedingungen der Produktion ableitet, ignoriert demgegenüber die eigenständige Bedeutung von textuellen Formen und Rezeptionsweisen.

Für Johnson ergeben sich aus seinem zirkulären Modell und aus der daraus abgeleiteten Kritik am Ökonomismus und Produktivismus - vor allem letzterer Vorwurf trifft auch die aktuelle Journalismusforschung in Deutschland - zwei Anforderungen an Produktionsanalysen: Zum einen müssen die Besonderheiten und eigenständigen Bedingungen der Produktion erfasst werden. Für die deutsche Journalismusforschung ist das im Rahmen der systemtheoretisch und konstruktivistisch argumentierenden Journalismusforschung schon weitgehend ausgearbeitet worden (vgl. Weischenberg 1992, 1995). Zum anderen jedoch müssen neben den redaktionellen, ökonomischen und institutionellen Bedingungen der Medienproduktion auch die kulturellen Voraussetzungen und Folgen der journalistischen Textproduktion thematisiert werden. Das erfordert eine Analyse der journalistischen Texte, ihrer Codes und Konventionen, die zugleich das Rohmaterial für journalistische Folgeproduktionen liefern.

Mit dem Kreislauf medialer Produktion und Rezeption wird sichtbar, dass die zentrale Erweiterung einer kulturorientierten Annäherung gegenüber den bislang in Deutschland dominierenden Forschungsansätzen in der Erweiterung um die Rezeptionsseite sowie die kontextuelle Einbindung der Rezipierenden ebenso wie der Produzierenden besteht. Die Abwendung von der vorrangigen Konzentration auf die Kommunikatoren und Journalistinnen und die verstärkte Hinwendung zum Publikum und seinen aktiven Deutungsleistungen der angebotenen Medientexte führt zwangsläufig zu einer Ausweitung des Journalismusbegriffs. Im Zentrum der Forschung stehen damit nicht allein Formen des Informations- und Nachrichtenjournalismus, sondern ebenso unterhaltsame, beratende, ironisierende, marktschreierische, erzählerische, boulevardeske, populäre Formen der Herstellung und Bereitstellung von Themen zur öffentlichen Kommunikation. Journalismus wird damit verstanden als kultureller Diskurs zur Selbstverständigung der Gesellschaft. Dieser Diskurs findet im Zusammenwirken von Produktion und Rezeption statt. Nur wenn Medientexte „erfolgreich“, also sinnverstehend rezipiert werden, nur dann können gesellschaftliche Übereinkünfte erzielt werden. 
Nur wenn die aktuelle journalistische Textproduktion an diese sinnhafte Dekodierung verfügbarer Textangebote anschließt, auf widerständige Deutungen Bezug nimmt und diese zum Gegenstand der Auseinandersetzung macht, kann Journalismus erfolgreich zur gesellschaftlichen Orientierung beitragen. Entlang der Elemente dieses Zirkels ergeben sich damit für die Journalismusforschung teils neue, teils veränderte Fragestellungen, die wir abschließend knapp skizzieren, um damit das Potenzial der Cultural Studies für die Journalistik sichtbar zu machen.

\subsection{Produktion}

Im Mittelpunkt der Erforschung der journalistischen Aussagenproduktion standen bislang das individuelle und redaktionelle Selbstverständnis sowie organisatorische Fragen der redaktionellen Selektionsprozesse und Entscheidungsstrukturen. Die von uns vorgeschlagene Perspektivierung rückt demgegenüber den diskursiven Prozess der Bedeutungszuweisung an diese Stelle. Zu fragen ist dabei nach dem Aushandeln von hegemonialer Bedeutung im Rahmen des redaktionellen Produktionsprozesses.

Am Beispiel der Berichterstattung über den CDU-Spendenskandal wäre also nachzuvollziehen, in welcher Weise die Auseinandersetzung zwischen der politischen Linie des Mediums, der individuellen Einschätzung der Redakteure und des gesellschaftlichen Diskurses außerhalb des Mediums zu grundsätzlichen Verschiebungen der parteipolitischen Orientierung überregionaler deutscher Tageszeitungen geführt haben. Entscheidend ist bei einer solchen Analyse nicht so sehr das „Was“ der politischen Positionsveränderung (die Demontage des politischen Übervaters Kohl), sondern das „Wie“ der Diskursgestaltung. Wie haben sich in die Medientexte Zweifel am Funktionieren der Parteiendemokratie eingeschrieben? Wie werden moralische Werte wie Aufrichtigkeit, Vertrauen, Zuverlässigkeit diskutiert?

Dabei erhalten die "Schreibweisen“ der Texte besondere Aufmerksamkeit. Sie können in Anlehnung an den Begriff der Lesarten in der Rezeption als Deutungsangebote verstanden werden, die sich in offenen Wertungen eines Ereignisses, in den dabei verwendeten Codes und Konventionen oder den im Text sichtbaren Kollektivsymbolen zeigen. Wie wird im Rahmen der Ressortbildung und der Aufgabenteilung zwischen den verschiedenen Medientypen ein gemeinsames kulturelles Wissen erzeugt? Zu analysieren ist dabei, welche Widersprüche in den Medien und im Redaktionsalltag zu veränderter Bedeutungsproduktion Anlass geben. Zu diesen Widersprüchen gehört heute, dass populäre Angebote ein großes Publikum erreichen, zugleich jedoch einer gesellschaftlichen Abwertung ausgesetzt sind. Die daran beteiligten JournalistInnen arbeiten in einer ambivalenten Positionierung zwischen populärer Wertschätzung und intellektueller Geringschätzung. ${ }^{8}$

Journalistische Textproduktionen sind als Ausdruck und Folge der Eingebundenheit von Journalisten und Redakteurinnen in eine spezifische gelebte Kultur, ein spezifisches

8 Ein Hinweis für diese widersprüchliche Positionierung lässt sich aus der Analyse des Publikumsbildes bei Scholl/Weischenberg (1998: 128 f.) entnehmen. Hier schreiben - für die Autoren kaum erklärlich - Journalisten in Medienorganisationen von geringer Komplexität und mit großer Reichweite dem Publikum stark staatsbürgerliche Eigenschaften zu. Dabei könnte es sich um einen Versuch handeln, der oben beschriebenen Ambivalenz von Wert- und Geringschätzung zu entkommen. Innerhalb einer kulturorientierten Forschung wäre dabei weniger die individuelle Beschreibung der JournalistInnen zu untersuchen als vielmehr die Frage, wie sich diese Ambivalenz und die Imaginationen des Publikums in die Medientexte einschreiben. 
Milieu zu untersuchen. Der Mediendiskurs ist nicht voraussetzungslos, sondern knüpft an Themen und Problematiken aus dem weiteren sozialen und politischen Umfeld der Kommunikatoren und Journalistinnen an. Das erfordert bei jeder Produktionsanalyse die Einbeziehung von Diskursmaterialien, die als Rohmaterial in die journalistischen Produkte einfließen.

Methodisch erfordern solche Untersuchungen des Produktionsprozesses Formen der teilnebmenden Beobachtung, worauf Rühl in einem der seltenen methodischen Beiträge zur Kommunikatorforschung bereits 1970 hingewiesen hat. Um zu beobachten und analytisch zu erfassen, wie im Rahmen der routinisierten journalistischen Arbeitsweise und der fest gefügten Medienorganisation Aushandlungsprozesse über kontextspezifische Bedeutungszuweisungen stattfinden, sind des Weiteren ethnografische Forschungsansätze Erfolg versprechend.

\subsection{Medientext}

Bei der Analyse der Bedeutungseinschreibung in journalistische Texte erscheinen in einem kulturorientierten Ansatz semiotische Mittel, Fragen der Mediendramaturgie sowie der Entwicklung von Genres von besonderer Relevanz. Fiske hat auf die Bedeutung der Textualität und Intertextualität von Fernsehtexten hingewiesen und ihnen in seinen verschiedenen Studien einen zentralen Platz eingeräumt. Hier liegen Ansatzpunkte zur Analyse journalistischer Produkte, deren Merkmale wie Auslassungen, Ironie, Übertreibung oder Exzess Produkt journalistischen Schreibens sind. Findet diese Art der Kodierung durch die Kommunikatoren bewusst statt oder ist er ein unbewusstes Ergebnis des Bemühens um eine „objektive“ Darstellung, die eindeutige Wertungen vermeidet? Erlauben solche textuellen Mittel eine Distanzierung von den Personen und Instanzen politischer Macht? Ermöglichen sie widersprüchliche und widerständige Bedeutungsproduktionen durch das Publikum? Welche Metaphern und Attribute enthalten hegemoniale Medientexte, an welche Bilder und historischen oder mythischen Vorstellungen knüpfen Medienakteure dabei an?

Intertextualität erscheint schließlich als zentraler Begriff, um die journalistischen Produkte in ihrem Bezug zu anderen Texten und in ihrer gesellschaftlichen Wirksamkeit zu untersuchen. Auf welche anderen medialen Produkte beziehen sich journalistische Texte, an welche zirkulierenden Bedeutungen knüpfen sie an, welche ignorieren sie? Welche Relevanz wird den einzelnen Texten innerhalb des Mediums und im Rahmen der Mediendramaturgie zugewiesen? Wie wird Relevanz und Aufmerksamkeit erzeugt und wie verändert das die Bedeutung der Texte? Wann vermitteln Text- und Bildelemente unterschiedliche, gar widersprüchliche Botschaften? Wie wird zwischen einzelnen Texten vermittelt und damit ein ganzheitlicher Bedeutungszusammenhang hergestellt? Welche Angebote werden dem Publikum zur selbstständigen dramaturgischen Gestaltung „seines“ Medienmenüs gemacht?

Schließlich gilt der Ausgestaltung und Entwicklung der Genres besondere Aufmerksamkeit. Auf der Ebene der Medientexte ist zu untersuchen, welche Bedeutungsangebote mit dem Entstehen neuer Darstellungsformen wie der Doku-Soap, dem Confrontainment oder allgemein verstärkt dialogischen Präsentationsformen (Talkshow, Doppelmoderation, Studiogespräch) verbunden sind. Welche Bedeutung haben diese journalistischen Genres, die an die mündliche Erzählkultur angelehnt und damit dem alltäglichen Sprachgebrauch stärker verhaftet sind als schriftliche und hochgradig standardisierte Genres wie Nachricht und Bericht? 
Methodisch sind hier also text- und diskursanalytische Verfabren gefragt, die die bestehenden inhaltsanalytischen Studien um semiotische oder linguistische Fragestellungen erweitern und weiter gehend nach der gesellschaftlichen Bedeutungsproduktion durch Journalismus fragen. Dazu liegen erste Ansätze vor (Bucher 2000; Fiske 1996; Jäger 2000).

\subsection{Rezeption}

JournalistInnen kodieren gesellschaftliche Ereignisse und stellen sie als Texte und Interpretationsangebote den RezipientInnen zur Verfügung. Dabei nehmen sie auf spezifische gelebte Kulturen Bezug, schreiben also mit Blick auf ein imaginiertes Publikum. Für die Frage nach dem Publikumsbezug ist dabei weniger das Image entscheidend, das individuelle JournalistInnen von ihrem diffusen Publikum haben, sondern die Frage, wie sie sich in der Verwendung von Sprache, Codes und Genres auf bestimmte Milieus beziehen.

Eine Antwort auf diese Frage erfordert den weiter gehenden Blick auf die Rezeptionsseite. Wie nutzen RezipientInnen die Polysemie der angebotenen Medientexte, in welcher Weise werden mediale Repräsentationen von Macht mit Bedeutung versehen? Aus der Rezeptionsanalyse fiktionaler Medientexte wissen wir, dass Vergnügen maßgeblich durch die u. U. widersprüchliche Dekodierung mehrdeutiger Textangebote entsteht. Lässt sich diese Erkenntnis auch auf die Rezeption journalistischer Texte übertragen? Mit Blick auf das sich verändernde Set an journalistischen Genres muss von der Rezeptionsseite her gefragt werden, wie unterschiedliche Genres identifiziert, mit Bedeutung versehen und funktional bestimmt werden. Wir haben deutliche Hinweise darauf, dass die funktionale Zuweisung von Nachrichten zum Zwecke der Information und Talkshows zum Zwecke der Unterhaltung von Rezipienten und Rezipientinnen durchbrochen und nach eigenen Kriterien neu zugewiesen wird (vgl. Dahlgren 1988; PausHaase u. a. 1999).

Von Interesse ist dabei vor allem, welche Funktion die jeweiligen Angebote für ihre RezipientInnen erhalten, welche Voraussetzungen sie an diese stellen, wie sie die Beteiligung an gesellschaftlichen Streitfragen ermöglichen oder erschweren. An welche Sprachcodes welcher gelebter Kulturen oder Teilöffentlichkeiten knüpfen sie dabei an (vgl. dazu die Untersuchung von Hamm / Koller 1992)? Erlauben diskursiv-dialogische stärker als monologisch-referierende Formen der Bedeutungsverhandlung in den Medien eine Integration des erworbenen „Wissens“ in das Alltagshandeln des Publikums? Davon ausgehend ist zu untersuchen, in welchem Maße die Publikumsaktivitäten auf den Journalismus zurückwirken, unter welchen Bedingungen wahrgenommene Publikumsreaktionen zu einer Veränderung journalistischer Produkte führen.

Methodisch bedient sich diese Perspektive der Instrumente der qualitativen Publikumsforschung, wobei das Focusgruppeninterview und die Beobachtung der (natürlichen) Rezeptionssituation (einschließlich der dabei produzierten sekundären Medientexte durch das unmittelbare Sprechen über den Medientext) einen besonderen Stellenwert einnehmen. Ergänzend können Formen des Medientagebuchs oder der Medienbiografie zum Einsatz kommen. 


\subsection{Schlussbemerkung}

„Personalisierung“, „Privatisierung“, „Fiktionalisierung“ und „Boulevardisierung“ sind konstitutive Merkmale journalistischer Produktion seitdem dieser dem Zeitgespräch der Gesellschaft eine Richtung gibt und es maßgeblich organisiert. Schon deshalb ist es notwendig, der Journalistik eine Blickrichtung zu geben, die diese Phänomene nicht als randständig für den Journalismus ansieht, sondern sie konzeptionell und methodisch integriert. Erst dann kann deutlich werden, was denn an der augenblicklichen Entwicklung das Neue und Andere ist und mit welchen Veränderungen in der Lebenswelt des Publikums sie korrespondiert.

\section{Literatur}

Allor, Marty and Madelaine Gagnon (1994): L'etat de culture. Genealogie discursive des politiques culturelles Quebecoises Montreal: Grecc (Concordia University/Universite de Montreal)

Bechdolf, Ute (1999): Puzzling Gender. Re- und Dekonstruktionen von Geschlechterverhältnissen im und beim Musikfernsehen. Weinheim

Blöbaum, Bernd (1994): Journalismus als soziales System. Geschichte, Ausdifferenzierung und Verselbständigung. Opladen

Böckelmann, Frank (1993): Journalismus als Beruf. Bilanz der Kommunikatorforschung im deutschsprachigen Raum von 1945 bis 1990. Konstanz

Bogart, Leo (1980): Television News as Entertainment. In: Tannenbaum, Percy H. (Hg.): The Entertainment Functions of Television. Hillsdale, S. $209-249$

Böning, Holger (1998): Das Private in der Aufklärung: Unterhaltung, Heirat, Tod in der Hamburger Presse, in den Intelligenzblättern und in der volksaufklärerischen Literatur und Publizistik des 18. Jahrhunderts. In: Imhof, Kurt; Schulz, Peter (Hg.), Die Veröffentlichung des Privaten Die Privatisierung des Öffentlichen, S. 45 - 54

Bosshart, Louis (1999): Media Entertainment. Prenote. In: Communication Research Trends 18, 3, S. $3-8$

Bromley, Roger (1999): Cultural Studies gestern und heute. In: Bromley, Roger; Göttlich, Udo; Winter, Carsten (Hg.): Cultural Studies. Grundlagentexte zur Einführung. Lüneburg, S. 9- 24

Bromley, Roger; Göttlich, Udo; Winter, Carsten (Hg.) (1999): Cultural Studies. Grundlagentexte zur Einführung. Lüneburg

Bruck, Peter A.; Stocker, Günther (1996): Die ganz normale Vielfältigkeit des Lesens. Zur Rezeption von Boulevardzeitungen. Münster

Bucher, Hans-Jürgen (2000): Journalismus als sprachliches Handeln. In: Löffelholz, Martin (Hg.): Theorien des Journalismus. Ein Text- und Studienbuch. Opladen/Wiesbaden (in Druck)

Corner, John (1991): Meaning, Genre and Context. The Problematics of „Public Knowledge“ in the New Audience Studies. In: Curran, James; Gurevitch, Michael (Hg.): Mass Media and Society. London et al., S. $267-284$

Dahlgren, Peter (1988): What's the Meaning of This? Viewer's Plural Sense-Making of TV News. In: Media, Culture and Society, 10, 3, S. $285-301$

Dahlgren, Peter (1992): Introduction. In: Dahlgren, Peter; Sparks, Colin (Hg.): Journalism and Popular Culture. London/Newbury Park/New Delhi, S. 1 - 23

Dehm, Ursula (1984): Fernseh-Unterhaltung. Zeitvertreib, Flucht oder Zwang? Eine sozialpsychologische Studie zum Fernseherleben. Mainz

Donohew, Lewis; Finn, Seth; Christ, William G. (1988): „The Nature of News“. Revisited: The Roles of Affect, Schemas, and Cognition. In: Donohew, Lewis; Sypher, Howard E.; Higgings, E. Tory (Hg.), Communication, Social Cognition and Affect. Hillsdale, S. $195-218$

Donsbach, Wolfgang (1982): Legitimationsprobleme des Journalismus. Freiburg/München

Donsbach, Wolfgang (1993): Redaktionelle Kontrolle im Jorunalimus. Eine internationaler Vergleich. In: Walter Mahle (Hg.): Journalisten in Deutschland. Nationale und internationale Vergleiche und Perspektiven. München, S. 143 - 160 
Donsbach, Wolfgang (1999): Journalism Research. In: Brosius, Hans-Bernd; Holtz-Bacha, Christina (Hg.): German Communication Yearbook. Cresskill, NJ, S. $159-180$

Esser, Frank (1998): Die Kräfte hinter den Schlagzeilen. Englischer und deutscher Journalismus im Vergleich. Freiburg, München

Ferguson, Marjorie; Golding, Peter (Hg.) (1997): Cultural Studies in Question. London et al.

Fiske, John (1989): Understanding Popular Culture. Boston

Fiske, John (1996): Media Matters. Race and Gender in U.S. Politics. Minneapolis/London

Görner, Felix (1995): Vom Außenseiter zum Aufsteiger. Ergebnisse der ersten repräsentativen Befragung von Sportjournalisten in Deutschland. Berlin

Göttlich, Udo; Winter, Carsten (1999): Wessen Cultural Studies? Zur Rezeption der Cultural Studies im deutschsprachigen Raum. In: Bromley, Roger; Göttlich, Udo; Winter, Carsten (Hg.): Cultural Studies. Grundlagentexte zur Einführung. Lüneburg, S. 25 - 39

Hall, Stuart (1973): Encoding and Decoding in the Television Discourse. Birmingham (= CCCS Stencilled Paper 7. University of Birmingham)

Hall, Stuart (1989): Die strukturierte Vermittlung von Ereignissen. In: Ausgewählte Schriften. Ideologie, Kultur, Medien, Neue Rechte, Rassismus. Hamburg, Berlin: Argument.

Hamm, Ingrid; Koller, Barbara (1992): Fernsehen und Wissensvermittlung. In: Winfried H. Schulz (Hg.): Medienwirkungen: Einflüsse von Presse, Radio und Fernsehen auf Individuum und Gesellschaft. Weinheim, S. $223-245$

Hartley, John (1996): Popular Reality. Journalism, Modernity, Popular Culture. London, New York, Sydney, Auckland

Hasebrink, Uwe; Krotz, Friedrich (1996): Die Zuschauer als Fernsehregisseure? Eine Einführung. In: dies. (Hg.): Die Zuschauer als Fernsehregisseure? Zum Verständnis individueller Nutzungs- und Rezeptionsmuster. Baden-Baden/Hamburg

Hepp, Andreas (1999): Cultural Studies und Medienanalyse. Eine Einführung. Opladen

Hepp, Andreas; Winter, Rainer (Hg.) (1997): Kultur - Medien - Macht. Cultural Studies und Medienanalyse. Opladen

Hermes, Joke (1997): Gender and Media Studies: no woman, no cry. In: John Corner, Philip Schlesinger and Roger Silverstone (eds.), International Handbook of Media Research. London, S. $65-95$

Hickethier, Knut (1997): Das Erzählen der Welt in den Fernsehnachrichten. Überlegungen zu einer Narrationstheorie der Nachricht. In: Rundfunk und Fernsehen, 45, S. 5 - 18

Jäckel, Michael; Peter, Jochen (1997): Cultural Studies aus kommunikationswissenschaftlicher Perspektive. Grundlagen und grundlegende Probleme. In: Rundfunk und Fernsehen, 45, S. 46-68

Jäger, Siegfried (2000): Kritische Diskursanalyse. Eine Einführung. 2. überarbeitete und erweiterte Auflage. Duisburg

Jensen, Klaus Bruhn (1988): News as Social Resource. A Qualitative Empirical Study of the Reception of Danish Television News. In: European Journal of Communication 3, S. $275-301$

Johnson, Richard (1985): Was ist überhaupt Kulturanalyse? In: Politische Sprachwissenschaft. Zur Analyse von Sprache als kulturelle Praxis. Hg. von Franz Januschek. Opladen, S. 23 - 69

Jürgs, Michael (1999): „Die viertklassige Gewalt“. In: Spiegel special: Info Sucht. Der Mensch im Netz der Medien. 3/1999, S. $64-66$

Klaus, Elisabeth (1996): Der Gegensatz von Information ist Desinformation, der Gegensatz von Unterhaltung ist Langeweile. In: Rundfunk und Fernsehen, 3/1996, S. $402-417$

Klaus, Elisabeth (1998): Kommunikationswissenschaftliche Geschlechterforschung. Zur Bedeutung der Frauen in den Massenmedien und im Journalismus. Opladen/Wiesbaden

Koch, Tom (1990): The News as Myth. Fact and Context in Journalism. New York/Westport, Connecticut/London

Krüger, Udo Michael (1996): Boulevardisierung der Information im Privatfernsehen. In: Media Perspektiven, 7, S. $362-374$

Langenbucher, Wolfgang R.; Mahle, Walter A. (1974): Unterhaltung als Beruf? Berlin

Langer, John (1998): Tabloid Television. Popular Journalism and the „Other News“. London/New York 
Lewis, Hustin (1994): The Absence of Narrative. Boredom and the Residual Power of Television News. In: Journal of Narrative and Life History, Special Issue, 4, 1 + 2, S. 25 - 40

Liebes, Tamar (1994): Narrativization of the News: An Introduction. In: Journal of Narrative and Life History, Special Issue, 4, 1+2, S. $1-8$

Lünenborg, Margret (2000): Europa ohne Öffentlichkeit? Theorien und Befunde europäischer Journalismusforschung. In: Martin Löffelholz (Hg.):; Theorien des Journalismus. Ein Textund Studienbuch. Opladen/Wiesbaden (im Druck)

Maar, Elke (1995): Bildung durch Unterhaltung. Die Entdeckung des Infotainment in der Aufklärung. Hallenser und Wiener Moralische Wochenschriften in der Blütezeit des Moraljournalismus, 1748 - 1782. Pfaffenweiler

Matthiesen, Sigrun (2000): Weiberkram. Wie der Kulturjournalismus mit der Mode umgeht. Wiesbaden

Merten, Klaus (1985): Faktoren der Rezeption von Nachrichtensendungen. Ergebnisbericht zum Projekt der ARD/ZDF-Medienkommission. Münster/Universität Münster

Merten, Klaus; Schmidt, Siegfried J.; Weischenberg, Siegfried (1994): Die Wirklichkeit der Medien. Eine Einführung in die Kommunikationswissenschaft. Opladen

Mettler-von Meibom, Barbara (1996): 10 Jahre dualer Rundfunk aus feministischer Sicht. Überlegungen zu einer notwendigen Diskussion. In: Hömberg, Walter; Pürer, Heinz (Hg.): MedienTransformation. Zehn Jahre dualer Rundfunk in Deutschland. Konstanz, S. 247 - 260

Morley, David (1980): The 'Nationwide' Audience. London: British Film Institute

Morley, David (1996): Medienpublika aus der Sicht der Cultural Studies. In: Hasebrink, Uwe; Krotz, Friedrich (Hg.): Die Zuschauer als Fernsehregisseure? Zum Verständnis individueller Nutzungs- und Rezeptionsmuster. Baden-Baden/Hamburg, S. 37 - 51

Neverla, Irene (1992): Fernseh-Zeit. Zuschauer zwischen Zeitkalkül und Zeitvertreib. Eine Untersuchung zur Fernsehnutzung. München

Paus-Haase, Ingrid; Hasebrink, Uwe; Mattusch, Uwe; Keunecke, Susanne; Krotz, Friedrich (1999): Daily Talks im Alltag von Jugendlichen. Schriftenreihe Medienforschung der Landesanstalt für Rundfunk Nordrhein-Westfalen, Bd. 32. Opladen

Patterson, Thomas; Donsbach, Wolfgang (1996): News Decisions. Journalists as Partisan Actors. In: Political Communication, 13, S. $455-468$

Renger, Rudi (1997): Spaß an „Information“. Journalismus als Populärkultur. In: Medien Journal 4, S. $23-40$

Renger, Rudi (2000): Populärer Journalismus. Bedeutungsproduktion und -rezeption zwischen Information und Unterhaltung". Salzburg (in Druck)

Rühl, Manfred (1970): Der Forscher als teilnehmender Beobachter der Arbeit und Organisation von Massenmedien. Probleme und Erfahrungen. In: Rundfunk und Fernsehen, 2, S. $156-168$

Rühl, Manfred (1980): Journalismus und Gesellschaft. Mainz

Rühl, Manfred (1993): Marktpublizistik. Oder: Wie alle - reihum - Presse und Rundfunk bezahlen. In: Publizistik 38, 2, S. $125-152$

Rühl, Manfred (2000): Theorien-Geschichte(n) des Journalismus. In: Löffelholz, Martin (Hg.): Theorien des Journalismus. Ein Text- und Studienbuch. Opladen/Wiesbaden (in Druck)

Ruhrmann, Georg (1989): Nachrichten und Rezipient. Struktur und Prozeß der Nachrichtenrekonstruktion. Opladen

Ruhrmann, Georg (1994): Ereignis, Nachricht und Rezipient. In: Merten, Klaus; Schmidt, Siegfried J.; Weischenberg, Siegfried (Hg.): Die Wirklichkeit der Medien. Eine Einführung in die Kommunikationswissenschaft. Opladen, S. $237-256$

Schmidt, Siegfied J.; Weischenberg, Siegfried (1994): Mediengattungen, Berichterstattungsmuster, Darstellungsformen. In: Merten, Klaus; Schmidt, Siegfried J.; Weischenberg, Siegfried, Die Wirklichkeit der Medien. Eine Einführung in die Kommunikationswissenschaft. Opladen, S. $212-326$

Schmitz, Manfred (1996): Märchen, Mythen und Symbole in massenmedialer Inszenierung. Zur Veränderung der Inhalte politischer Kommunikation. In: medium 26, 1, S. 46 - 50

Schneider, Beate; Schönbach, Klaus; Stürzebecher, Dieter (1993a): Westdeutsche Journalisten im 
Vergleich: jung, professionell und mit Spaß an der Arbeit. In: Publizistik, 38, 1, S. 5 - 30

Schneider, Beate; Schönbach, Klaus; Stürzebecher, Dieter (1993b): Journalisten im vereinigten Deutschland. Strukturen, Arbeitsweisen und Einstellungen im Ost-West-Vergleich. In: Publizistik, 38, 1, S. $353-382$

Schneider, Beate; Schönbach, Klaus; Stürzebecher, Dieter (1994): II. Ergebnisse einer Repräsentativbefragung zur Struktur, sozialen Lage und zu den Einstellungen von Journalisten in den neuen Bundesländern. In: Böckelmann, Frank; Mast, Claudia; Schneider, Beate (Hg.): Journalismus in den neuen Ländern. Ein Berufsstand zwischen Aufbruch und Abwicklung. Konstanz, S. $143-230$

Scholl, Armin (1997): Journalismus als Gegenstand empirischer Forschung: Ein Definitionsvorschlag. In: Publizistik, 42, 4, S. 468 - 486

Scholl, Armin; Weischenberg, Siegfried (1998): Journalismus in der Gesellschaft. Theorie, Methodologie und Empirie. Opladen/Wiesbaden

Sievert, Holger (1998): Europäischer Journalismus. Theorie und Empirie aktueller Medienkommunikation in der Europäischen Union. Opladen/Wiesbaden

Sparks, Colin (1992): Popular Journalism: Theories and Practice. In: Dahlgren, Peter; Sparks, Colin (Hg.): Journalism and Popular Culture. London et al., S. $24-44$

Starkulla, Heinz (1963): Publizistik und Kommunikation. In: Festschrift für Hanns Braun. Bremen

Teichert, Will (1987): Die ausdruckslosen Bilder müssen für jeden Text verwendbar sein. In: Frankfurter Rundschau, Nr. 97 vom 27.4.1987, S. 10

Weaver, David H.; Wilhoit, G. Cleveland (1996): The American Journalist in the 1990s. U.S. News People at the End of an Era. Mahwah

Weischenberg, Siegfried (1992): Journalistik. Theorie und Praxis aktueller Medienkommunikation, Bd. 1: Mediensysteme, Medienethik, Medieninstitutionen. Opladen

Weischenberg, Siegfried (1995): Journalistik. Theorie und Praxis aktueller Medienkommunikation, Bd. 2: Medientechnik, Medienfunktionen, Medienakteure. Opladen

Winter, Rainer (1997): Cultural Studies als kritische Medienanalyse. Vom „encoding/decoding“ Modell zur kritischen Medienanalyse. In: Hepp, Andreas; Winter, Rainer (Hg.): Kultur - Medien - Macht. Cultural Studies und Medienanalyse. Opladen, S. 47 - 63

Zoonen, Liesbet van (1994): Feminist Media Studies. London/Thousand Oaks/New Delhi 